Research Article

\title{
Iterative Scheme for Split Variational Inclusion and a Fixed-Point Problem of a Finite Collection of Nonexpansive Mappings
}

\author{
M. Dilshad $\mathbb{D}^{1}{ }^{1}$ A. F. Aljohani, ${ }^{1}$ and M. Akram ${ }^{2}$ \\ ${ }^{1}$ Department of Mathematics, Faculty of Science, University of Tabuk, Tabuk 71491, Saudi Arabia \\ ${ }^{2}$ Department of Mathematics, Faculty of Science, Islamic University of Madinah, Saudi Arabia \\ Correspondence should be addressed to M. Dilshad; mdilshaad@gmail.com
}

Received 31 July 2020; Revised 22 August 2020; Accepted 31 August 2020; Published 9 October 2020

Academic Editor: Mohammad Mursaleen

Copyright (c) 2020 M. Dilshad et al. This is an open access article distributed under the Creative Commons Attribution License, which permits unrestricted use, distribution, and reproduction in any medium, provided the original work is properly cited.

\begin{abstract}
This article is aimed at introducing an iterative scheme to approximate the common solution of split variational inclusion and a fixed-point problem of a finite collection of nonexpansive mappings. It is proven that under some suitable assumptions, the sequences achieved by the proposed iterative scheme converge strongly to a common element of the solution sets of these problems. Some consequences of the main theorem are also given. Finally, the convergence analysis of the sequences achieved from the iterative scheme is illustrated with the help of a numerical example.
\end{abstract}

\section{Introduction}

Let $\mathscr{H}_{1}$ and $\mathscr{H}_{2}$ be two real Hilbert spaces endowed with inner product $\langle\cdot, \cdot\rangle$ and induced norm $\|\cdot\|$. A mapping $T: \mathscr{H}_{1} \longrightarrow \mathscr{H}_{1}$ is called contraction, if $\exists \kappa \in(0,1)$ such that $\|T(\varphi)-T(\psi)\| \leq \kappa\|\varphi-\psi\|, \forall \varphi, \psi \in \mathscr{H}_{1}$. If $\kappa=1$, then $T$ becomes nonexpansive. A mapping $T$ is said to have a fixed point, if $\exists \varphi \in\left(\mathscr{H}_{1}\right)$ such that $T(\varphi)=(\varphi)$. Further, if $T_{n}: \mathscr{H}_{1} \longrightarrow \mathscr{H}_{1}, \quad(n=1, \cdots, M)$ is a finite collection of nonexpansive mappings. Then, the fixed-point problem (FPP) is defined as find $\varphi \in \mathscr{H}_{1}$ such that

$$
\bigcap_{n=1}^{M} T_{n}(\varphi)=\varphi
$$

It is easy to show that if $\bigcap_{n=1}^{M} \operatorname{Fix}\left(T_{n}\right) \neq 0$, then $\bigcap_{n=1}^{M}$ $\operatorname{Fix}\left(T_{n}\right)$ is closed and convex. Many iterative methods have been adopted to examine the solution of a fixedpoint problem for nonexpansive mappings and its variant forms, see [1-5] and references therein.
We know that most of the techniques for solving the fixed-point problems can be acquired from Mann's iterative technique [3], namely, for arbitrary $x_{0} \in \mathscr{C}$, compute

$$
x_{k+1}=\alpha_{k} x_{k}+\left(1-\alpha_{k}\right) T x_{k}, \quad k \geq 0,
$$

where $T$ is a nonexpansive mapping from a nonempty closed convex subset $\mathscr{C}$ of Hilbert space $\mathscr{H}_{1}$ to itself and $\alpha_{n}$ is a control sequence, which force $\left\{x_{k}\right\}$ to converge (weak) to a fixed point of $T$. To obtain the strong convergence result, Moudafi [4] proposed the viscosity approximation method by combining the nonexpansive mapping $T$ with a contraction of given mapping $f$ over $\mathscr{C}$. For an arbitrary $x_{0} \in \mathscr{C}$, compute the sequence $\left\{x_{k}\right\}$ generated by

$$
x_{k+1}=\alpha_{k} f\left(x_{k}\right)+\left(1-\alpha_{k}\right) T x_{k}, \quad k \geq 0,
$$

where $\alpha_{n} \in(0,1)$ goes slowly to zero. The sequence $\left\{x_{k+1}\right\}$ achieved from this iterative method converges strongly to a fixed point of $T$.

On the other hand, let us recall some work about split variational inequality/inclusion problems. A multivalued 
mapping $G: \mathscr{H}_{1} \longrightarrow 2^{\mathscr{H}_{1}}$ is called maximal monotone, if its graph $\operatorname{gph}(G)=\left\{(\varphi, \psi) \in \mathscr{H}_{1} \times \mathscr{H}_{1}: \psi \in G(\varphi)\right\}$ is not properly contained by the graph of any other monotone mapping. A monotone mapping $G$ is maximal monotone if and only if for $(\varphi, \zeta) \in \mathscr{H}_{1} \times \mathscr{H}_{1},\langle\varphi-\psi, \zeta-\vartheta\rangle \geq 0$ for every $(\psi, \vartheta) \epsilon$ $\operatorname{gph}(G)$ implies that $\zeta \in G(\varphi)$. If $G$ is maximal monotone, then operator $J_{\lambda}^{G}=(I+\lambda G)^{-1}$ is well defined, nonexpansive, and known as the resolvent of $G$ with parameter $\lambda>0$, which is defined at every point of the domain.

The idea of split variational inequality problem (SVIP) given by Censor et al. [6], which amounts to saying find a solution of variational inequality whose image, under a given bounded linear operator, solves another variational inequality. Find $\varphi^{*} \in \mathscr{C}$ such that

$$
\left\langle h\left(\varphi^{*}\right), \varphi-\varphi^{*}\right\rangle \geq 0, \quad \forall \varphi \in \mathscr{C},
$$

and such that

$$
\psi^{*}=A \varphi^{*} \in \mathscr{D} \text { solves }\left\langle g\left(\psi^{*}\right), \psi-\psi^{*}\right\rangle \geq 0, \quad \forall \psi \in \mathscr{D},
$$

where $\mathscr{C}$ and $\mathscr{D}$ are closed, convex subsets of Hilbert spaces $\mathscr{H}_{1}$ and $\mathscr{H}_{2}$, respectively; $A: \mathscr{H}_{1} \longrightarrow \mathscr{H}_{2}$ is a bounded linear operator, and $h: \mathscr{H}_{1} \longrightarrow \mathscr{H}_{1}$ and $g: \mathscr{H}_{2} \longrightarrow \mathscr{H}_{2}$ are two operators. They studied the weak convergent result to solve SVIP.

Moudafi [7] generalized SVIP and introduced split monotone variational inclusion problem ( $\left.\mathrm{S}_{\mathrm{p}} \mathrm{MVIP}\right)$ : find $\varphi^{*} \in \mathscr{H}_{1}$ such that

$$
0 \in h\left(\varphi^{*}\right)+G_{1}\left(\varphi^{*}\right)
$$

and such that

$$
\psi^{*}=A \varphi^{*} \in \mathscr{H}_{2} \text { solves } 0 \in g\left(\psi^{*}\right)+G_{2}\left(\psi^{*}\right) \text {, }
$$

where $G_{1}: \mathscr{H}_{1} \longrightarrow 2^{\mathscr{H}_{1}}$ and $G_{2}: \mathscr{H}_{2} \longrightarrow 2^{\mathscr{H}_{2}}$ are multivalued monotone mappings, $A: \mathscr{H}_{1} \longrightarrow \mathscr{H}_{2}$ is a bounded linear operator, $h: \mathscr{H}_{1} \longrightarrow \mathscr{H}_{1}$ and $g: \mathscr{H}_{2} \longrightarrow \mathscr{H}_{2}$ are two single-valued operators. The author also composed an iterative algorithm to solve ( $S_{p} M V I P$ ) and showed that the sequence achieved by the proposed algorithm converges weakly to the solution of $\left(\mathrm{S}_{\mathrm{p}} \mathrm{MVIP}\right)$. Numerous iterative methods have been investigated for split variational inequality/inclusion problems, split common fixed-point problems, split feasibility problems, and split zero problems and their generalizations, see $[6,8-16]$ and references therein.

If $h=g=0$ in S MVIP, then we obtain the split variational inclusion problem $\left(\mathrm{S}_{\mathrm{p}} \mathrm{VIP}\right)$ considered in [8], stated as find $\varphi^{*} \in \mathscr{H}_{1}$ such that

$$
0 \in G_{1}\left(\varphi^{*}\right)
$$

such that

$$
\psi^{*}=A \varphi^{*} \in \mathscr{H}_{2} \text { solves } 0 \in G_{2}\left(\psi^{*}\right)
$$

Byrne et al. [8] proposed the following iterative scheme for $\mathrm{S}_{\mathrm{p}}$ VIP and studied the strong and weak convergence. For arbitrary $x_{o} \in \mathscr{H}_{1}$, compute the iterative sequence achieved by the following scheme:

$$
x_{k+1}=J_{\lambda}^{G_{1}}\left[\left(x_{k}+\mu A^{*}\left(J_{\lambda}^{G_{2}}-I\right) A x_{k}\right)\right]
$$

for $\lambda>0$.

Recently, Kazmi and Rizvi [17] suggested and examined an iterative algorithm to estimate the common solution for $\mathrm{S}_{\mathrm{p}}$ VIP and a fixed-point problem of a nonexpansive mapping in Hilbert spaces. Puangpee and Sauntai [18] studied the split variational inclusion problem and fixed-point problem in Banach spaces. Haitao and $\mathrm{Li}$ [19] investigated the split variational inclusion problem and fixed-point problem of nonexpansive semigroup without prior calculation of operator norm. Later, many authors studied the common solution of split variational inequality/inclusion problem and fixedpoint problem of nonexpansive mappings in the framework of Hilbert/Banach spaces, see for example [18-24] and references therein.

Following the works in $[4,7,8,17]$ and by the current research in this flow, we propose an iterative scheme to approximate a common solution of FPP and $\mathrm{S}_{\mathrm{p}} \mathrm{VIP}$. We prove that the sequences achieved by the proposed iterative scheme strongly converge to the common solution of FPP and $S_{p}$ VIP. The iterative scheme and results discussed in this article are new and can be viewed as generalization and refinement of the previously published work in this area.

\section{Prelude and Auxiliary Results}

In this section, we assembled some underlying definitions and supporting results.

Definition 1. Let $\mathscr{C}\left(\mathscr{C} \subset \mathscr{H}_{1}\right)$, the metric projection $P_{\mathscr{C}}$ onto the set $\mathscr{C}$ is defined as $P_{\mathscr{C}}(\vartheta) \in \mathscr{C}$ and $\left\|\vartheta-P_{\mathscr{C}}(\vartheta)\right\|=$ $\inf _{\vartheta \in \mathscr{C}}\|\vartheta-\omega\|, \forall \omega \in \mathscr{H}_{1}$.

$P_{\mathscr{C}}$ is also characterised by the facts that $P_{\mathscr{C}}(\vartheta) \in \mathscr{C}$,

$$
\left\langle\vartheta-P_{\mathscr{C}}(\vartheta), \omega-P_{\mathscr{C}}(\vartheta)\right\rangle \leq 0
$$

and

$$
\|\vartheta-\omega\|^{2} \geq\left\|\vartheta-P_{\mathscr{C}}(\vartheta)\right\|^{2}+\left\|\omega-P_{\mathscr{C}}(\vartheta)\right\|^{2}, \quad \forall \vartheta \in \mathscr{H}_{1}, \omega \in \mathscr{C}
$$

Remark 2 (see $[25,26])$. For a nonexpansive mapping $T$ and projection $P_{\mathscr{C}}(\vartheta)$ onto $\mathscr{C}$, the following results hold in Hilbert spaces: 
(i)

$$
\left\langle\mathcal{Y}-\omega, P_{\mathscr{C}}(\mathcal{Y})-P_{\mathscr{C}}(\omega)\right\rangle \geq\left\|P_{\mathscr{C}}(\mathcal{Y})-P_{\mathscr{C}}(\omega)\right\|^{2}, \quad \forall \mathcal{Y}, \omega \in \mathscr{H}_{1}
$$

(ii) For all $(\vartheta, \omega) \in \mathscr{H}_{1} \times \mathscr{H}_{1}$,

$$
\begin{gathered}
\langle(\vartheta-T(\vartheta))-(\omega-T(\omega)), T(\omega)-T(\vartheta)\rangle \\
\quad \leq \frac{1}{2}\|(T(\vartheta)-\vartheta)-(T(\omega)-(\omega))\|^{2} .
\end{gathered}
$$

Thus, for all $(\vartheta, \omega) \in \mathscr{H}_{1} \times \operatorname{Fix}(T)$, we get

$$
\langle\vartheta-T(\vartheta), \omega-T(\omega)\rangle \leq \frac{1}{2}\|T(\vartheta)-\vartheta\|^{2}
$$

(iii) For all $\vartheta, \omega \in \mathscr{H}_{1}, t \in(0,1)$

$$
\begin{aligned}
\|t \vartheta-(1-t) \omega\|^{2}= & t\|\vartheta\|^{2}+(1-t)\|\omega\|^{2} \\
& -t(1-t)\|\vartheta-\omega\|^{2}
\end{aligned}
$$

Definition 3. A mapping $T: \mathscr{H}_{1} \longrightarrow \mathscr{H}_{1}$ is said to be

(i) monotone, if

$$
\langle T \vartheta-T \omega, \vartheta-\omega\rangle \geq 0, \quad \text { for all } \vartheta, \omega \in \mathscr{H}_{1}
$$

(ii) $\tau$-strongly monotone, if there exists a constant $\tau>0$ such that

$$
\langle T \vartheta-T \omega, \vartheta-\omega\rangle \geq \tau\|\vartheta-\omega\|^{2}, \quad \forall \vartheta, \omega \in \mathscr{H}_{1}
$$

(iii) $\gamma$-inverse strongly monotone, if there exists a constant $\gamma>0$ such that

$$
\langle T \vartheta-T \omega, \vartheta-\omega\rangle \geq \gamma\|T \vartheta-T \omega\|^{2}, \quad \forall \vartheta, \omega \in \mathscr{H}_{1}
$$

(iv) firmly nonexpansive, if

$$
\langle T \vartheta-T \omega, \vartheta-\omega\rangle \geq\|T \vartheta-T \omega\|^{2}, \quad \forall \vartheta, \omega \in \mathscr{H}_{1}
$$

Some important characteristics of an averaged operator are mentioned below; for more details, we refer to $[7,27,28]$.

Definition 4. A mapping $T: \mathscr{H}_{1} \longrightarrow \mathscr{H}_{1}$ is called an averaged if and only if $T$ is the average of identity mapping and a nonexpansive mapping, that is, $T=(1-t) I+t S$, where $t \in(0,1)$ and $S: \mathscr{H}_{1} \longrightarrow \mathscr{H}_{1}$ is nonexpansive.
Thus, firmly nonexpansive mappings are averaged. It can also be seen that averaged mappings are nonexpansive.

\section{Proposition 5.}

(i) Let $S: \mathscr{H}_{1} \longrightarrow \mathscr{H}_{1}$ be an averaged and $V: \mathscr{H}_{1} \longrightarrow$ $\mathscr{H}_{1}$ be a nonexpansive mapping, then $T=(1-t) S+$ $t V$ is averaged for $t \in(0,1)$

(ii) If the composite $\left\{T_{n}\right\}_{n=1}^{M}$ is averaged and have a nonempty common fixed point, then

$$
\bigcap_{n=1}^{M} \operatorname{Fix}\left(T_{n}\right)=\operatorname{Fix}\left(T_{1} T_{2} \cdots \cdots \cdots T_{M}\right)
$$

(iii) If $T$ is $\gamma$-inverse strongly monotone, then for $r>0, r T$ is $r / \gamma$-inverse strongly monotone

(iv) $T$ is averaged if its compliment $I-T$ is $\gamma$-inverse strongly monotone for some $\gamma>(1 / 2)$

Lemma 6 (see [29]). Assume that $T$ is nonexpansive selfmapping of a closed convex subset $\mathscr{D}$ of a Hilbert space $\mathscr{H}_{1}$. If $T$ has a fixed point, then $I-T$ is demiclosed, i.e., whenever $\left\{\omega_{n}\right\}$ is a sequence in $\mathscr{D}$ converging weakly to some $\omega \in \mathscr{D}$ and the sequence $\left\{(I-T) \omega_{n}\right\}$ converges strongly to some $\omega$, then $(I-T) \omega=\varrho$, where I is the identity mapping on $\mathscr{H}_{1}$.

Lemma 7 (see [5]). If $\left\{v_{k}\right\}$ is a sequence of nonnegative real numbers such that

$$
v_{k+1} \leq\left(1-\xi_{n}\right) v_{k}+\omega_{k}, \quad k=0,1,2 \cdots
$$

where $\left\{\xi_{k}\right\}$ is a sequence in $(0,1)$ and $\left\{\omega_{k}\right\}$ is a sequence in $\mathbb{R}$ such that

(i) $\sum_{k=1}^{\infty} \xi_{k}=\infty$

(ii) $\lim \sup _{k \rightarrow \infty}\left(\omega_{k} / \xi_{k}\right) \leq 0$ or lim $\sup _{k \rightarrow \infty}\left|\omega_{k}\right|<\infty$

Then, $\lim _{k \rightarrow \infty} v_{k}=0$.

We denote the solution set of $S_{p}$ VIP by $\Xi=\left\{\varphi^{*} \in \mathscr{H}_{1}\right.$ : $0 \in G_{1}\left(\varphi^{*}\right)$ and $\left.0 \in G_{2}\left(A \varphi^{*}\right)\right\}$ and of FPP by $\cap_{n=1}^{M} \operatorname{Fix}\left(T_{n}\right)$.

\section{Iterative Scheme and Its Convergence}

In this section, we present the iterative scheme and show that the sequences obtained from the proposed iterative scheme converge strongly to the common solution of FPP and $\mathrm{S}_{\mathrm{p}} \mathrm{VIP}$.

For integer $K \geq 1$, we define the mapping $T_{[K]}=T_{K \bmod M}$ with the mod function, which is taking values from the set $\{1,2, \cdots, M\}$, that is, if $K=a M+b$ for some integer $a \geq 0$ and $0 \leq b \leq M$, then $T_{[K]}=T_{M}$ if $b=0$ and $T_{[K]}=T_{b}$ if $0<b<M$.

Iterative Scheme 8. Step 0. Take $\left\{\alpha_{k}\right\} \subset(0,1)$. Choose $u_{0} \in$ $\mathscr{H}_{1}$ arbitrary and let $k=0$. 
Step 1. Given $u_{k} \in \mathscr{H}_{1}$, compute $u_{k+1} \in \mathscr{H}_{1}$ as

$$
\begin{aligned}
v_{k} & =J_{\lambda}^{G_{1}}\left[u_{k}+\mu A^{*}\left(J_{\lambda}^{G_{2}}-I\right) A u_{k}\right], \\
u_{k+1} & =\alpha_{k} f\left(u_{k}\right)+\left(1-\alpha_{k}\right) T_{[k+1]} v_{k},
\end{aligned}
$$

update $k=k+1$ and go to Step 1 .

Condition $C$. We assume that $T_{n}: \mathscr{H}_{1} \longrightarrow \mathscr{H}_{1},(n=1,2, \cdots$, $M)$, is a finite number of nonexpansive mappings such that $\cap_{n=1}^{M} \operatorname{Fix}\left(T_{n}\right) \neq 0$ and

$$
\begin{aligned}
\bigcap_{n=i}^{M} \operatorname{Fix}\left(T_{n}\right) & =\operatorname{Fix}\left(T_{1} \circ T_{2} \circ \cdots \circ T_{M}\right)=\operatorname{Fix}\left(T_{M} \circ T_{1} \circ \cdots \circ T_{M-1}\right) \\
& =\cdots=\operatorname{Fix}\left(T_{2} \circ T_{3} \circ \cdots \circ T_{M} \circ T_{1}\right) .
\end{aligned}
$$

Lemma 9. $\varphi^{*} \in \mathscr{H}_{1}$ and $\psi^{*}=A \varphi^{*}$ are solutions of $S_{p} V I P$, if and only if $\varphi^{*}=J_{\lambda}^{G_{1}}\left(\varphi^{*}\right)$ and $\psi^{*}=A \varphi^{*}=J_{\lambda}^{G_{2}}\left(\psi^{*}\right)$, for some $\lambda>0$.

Proof. The proof of the lemma follows immediately from the definitions of resolvent operators.

Remark 10. If $J_{\lambda}^{G}$ is the resolvent of maximal monotone mapping $G, A^{*}$ is the adjoint operator of $A$ and $\mathscr{R}$ is the spectral radius of $A A^{*}$. Then, using the properties of averaged mapping, one can easily show that the operator $\left[I+\mu A^{*}\left(J_{\lambda}^{G}-I\right) A\right]$ is averaged with $\lambda>0, \mu \in(0,1 / \mathscr{R})$.

Now, we prove the following lemma which guarantees the contractivity of $L$, which is needed to prove our main result.

Lemma 11. Let $\mathscr{H}_{1}$ and $\mathscr{H}_{2}$ be two real Hilbert spaces and $A: \mathscr{H}_{1} \longrightarrow \mathscr{H}_{2}$ be a bounded linear operator. Suppose that $G_{1}: \mathscr{H}_{1} \longrightarrow \mathscr{H}_{1}$ and $G_{2}: \mathscr{H}_{2} \longrightarrow \mathscr{H}_{2}$ are maximal monotone operators and $T: \mathscr{H}_{1} \longrightarrow \mathscr{H}_{1}$ be a nonexpansive mapping. Let $f: \mathscr{H}_{1} \longrightarrow \mathscr{H}_{1}$ be a $\kappa$-contraction mapping with constant $\kappa>0$. For any $\theta \in(0,1]$, we define a mapping on $\mathscr{H}_{1}$ by

$L(\vartheta)=\theta f(\vartheta)+(1-\theta) T\left[J_{\lambda}^{G_{1}}\left(\vartheta+\mu A^{*}\left(J_{\lambda}^{G_{2}}-I\right) A \vartheta\right)\right], \quad \forall \vartheta \in \mathscr{H}_{1}$,

where $\mu \in(0,1 / \mathscr{R}), \mathscr{R}$ is the spectral radius of the operator $A A^{*}$, and $A^{*}$ is the adjoint operator of $A$. Then, the mapping $L$ is a contraction with constant $0<1-\theta(1-\kappa)<1$; hence, $L$ has a unique fixed point.

Proof. The operators $J_{\lambda}^{G_{1}}$ and $J_{\lambda}^{G_{2}}$ are averaged being firmly nonexpansive. For $\mu \in(0,1 / \mathscr{R})$, the operators
$\left[I+\mu A^{*}\left(J_{\lambda}^{G_{2}}-I\right) A\right]$ and $J_{\lambda}^{G_{1}}\left(I+\mu A^{*}\left(J_{\lambda}^{G_{2}}-I\right) A\right)$ are averaged and hence nonexpansive. Thus, for all $u, v \in \mathscr{H}_{1}$, we have

$$
\begin{aligned}
&\|L(\vartheta)-L(\omega)\| \\
&= \| \theta f(\vartheta)+(1-\theta) T\left[J_{\lambda}^{G_{1}}\left(\vartheta+\mu A^{*}\left(J_{\lambda}^{G_{2}}-I\right) A \vartheta\right)\right] \\
& \quad-\theta f(\omega)-(1-\theta) T\left[J_{\lambda}^{G_{1}}\left(\omega+\mu A^{*}\left(J_{\lambda}^{G_{2}}-I\right) A \omega\right)\right] \| \\
& \leq \theta\|f(\vartheta)-f(\omega)\|+(1-\theta)\|T \vartheta-T \omega\| \\
& \leq \theta \kappa\|\vartheta-\omega\|+(1-\theta)\|\vartheta-\omega\| \\
&= {[1-\theta(1-\kappa)]\|\vartheta-\omega\| . }
\end{aligned}
$$

Since $0<1-\theta(1-\kappa)<1$ implies that $L$ is a contraction, hence $L$ has a unique fixed point.

Theorem 12. Let $\mathscr{H}_{1}$ and $\mathscr{H}_{2}$ be two real Hilbert spaces and A : $\mathscr{H}_{1} \longrightarrow \mathscr{H}_{2}$ be a bounded linear operator. Assume that $G_{1}: \mathscr{H}_{1} \longrightarrow 2^{\mathscr{H}_{1}}$ and $G_{2}: \mathscr{H}_{2} \longrightarrow 2^{\mathscr{H}_{2}}$ are two maximal monotone operators and $f: \mathscr{H}_{1} \longrightarrow \mathscr{H}_{1}$ is a contraction with constant $\kappa \in(0,1)$. Let $T_{n}: \mathscr{H}_{1} \longrightarrow \mathscr{H}_{1},(n=1,2, \cdots, M)$, be a finite collection of nonexpansive mappings satisfying the Condition $C$ such that $\cap_{n=1}^{M} F i x\left(T_{n}\right) \cap \Xi \neq 0$. Let $\mathscr{R}$ be a spectral radius of $A^{\star} A$, where $A^{\star}$ is the adjoint of $A$ such that $\mu \in(0,1 / \mathscr{R})$ and $\left\{\alpha_{k}\right\}$ be a sequence in $(0,1)$ such that $\lim _{k \rightarrow \infty}$ $\alpha_{k}=0, \sum_{k=1}^{\infty} \alpha_{k}=\infty$, and $\sum_{k=1}^{\infty}\left|\alpha_{k}-\alpha_{k-1}\right|<\infty$. Then, the iterative sequences $\left\{v_{k}\right\}$ and $\left\{u_{k}\right\}$ generated by Iterative Scheme 8 converge strongly to $\bar{v} \in \cap_{n=1}^{M} \operatorname{Fix}\left(T_{n}\right) \cap \Xi$, where $\bar{v}=P_{\cap_{n=1}^{M} F i x\left(T_{n}\right) \cap \Xi} f(\bar{v})$.

Proof. Let $u^{\star} \in \cap_{n=1}^{M} \operatorname{Fix}\left(T_{n}\right) \cap \Xi$, then we have $J_{\lambda}^{G_{1}} u^{\star}=u^{\star}$, $J_{\lambda}^{G_{2}} A u^{\star}=A u^{\star}$, and $T_{n}\left(u^{\star}\right)=u^{\star},(n=1,2, \cdots M)$, then using Iterative Scheme 8 , we evaluate

$$
\begin{aligned}
\left\|v_{k}-u^{\star}\right\|^{2}= & \left\|J_{\lambda}^{G_{1}}\left(u_{k}+\mu A^{*}\left(J_{\lambda}^{G_{2}}-I\right) A u_{k}\right)-u^{\star}\right\|^{2} \\
= & \left\|J_{\lambda}^{G_{1}}\left(u_{k}+\mu A^{*}\left(J_{\lambda}^{G_{2}}-I\right) A u_{k}\right)-J_{\lambda}^{G_{1}} u^{\star}\right\|^{2} \\
\leq & \left\|u_{k}-u^{\star}\right\|^{2}+\mu^{2}\left\|A^{\star}\left(J_{\lambda}^{G_{2}}-I\right) A u_{k}\right\|^{2} \\
& +2 \mu\left\langle u_{k}-u^{\star}, A^{*}\left(J_{\lambda}^{G_{2}}-I\right) A u_{k}\right\rangle \\
= & \left\|u_{k}-u^{\star}\right\|^{2}+\mu^{2}\left\langle\left(J_{\lambda}^{G_{2}}-I\right) A u_{k}, A A^{*}\left(J_{\lambda}^{G_{2}}-I\right) A u_{k}\right\rangle \\
& +2 \mu\left\langle u_{k}-u^{\star}, A^{*}\left(J_{\lambda}^{G_{2}}-I\right) A u_{k}\right\rangle \\
\leq & \left\|u_{k}-u^{\star}\right\|^{2}+\mu^{2} \mathscr{R}\left\|\left(J_{\lambda}^{G_{2}}-I\right) A u_{k}\right\|^{2} \\
& +2 \mu\left\langle u_{k}-u^{\star}, A^{*}\left(J_{\lambda}^{G_{2}}-I\right) A u_{k}\right\rangle .
\end{aligned}
$$

Denoting $\nabla=2 \mu\left\langle u_{k}-u^{\star}, A^{*}\left(J_{\lambda}^{G_{2}}-I\right) A u_{k}\right\rangle$ and using (16), we have 


$$
\begin{aligned}
\nabla= & 2 \mu\left\langle A\left(u_{k}-u^{\star}\right),\left(J_{\lambda}^{G_{2}}-I\right) A u_{k}\right\rangle \\
= & 2 \mu\left\langle A\left(u_{k}-u^{\star}\right)+\left(J_{\lambda}^{G_{2}}-I\right) A u_{k}\right. \\
& \left.-\left(J_{\lambda}^{G_{2}}-I\right) A u_{k},\left(J_{\lambda}^{G_{2}}-I\right) A u_{k}\right\rangle \\
= & 2 \mu\left\{\left\langle J_{\lambda}^{G_{2}} A u_{k}-A u^{\star},\left(J_{\lambda}^{G_{2}}-I\right) A u_{k}\right\rangle-\left\|\left(J_{\lambda}^{G_{2}}-I\right) A u_{k}\right\|^{2}\right\} \\
\leq & 2 \mu\left\{\frac{1}{2}\left\|\left(J_{\lambda}^{G_{2}}-I\right) A u_{k}\right\|^{2}-\left\|\left(J_{\lambda}^{G_{2}}-I\right) A u_{k}\right\|^{2}\right\} \\
\leq & -\mu\left\|\left(J_{\lambda}^{G_{2}}-I\right) A u_{k}\right\|^{2},
\end{aligned}
$$

using (28), (27) becomes

$$
\left\|v_{k}-u^{\star}\right\|^{2} \leq\left\|u_{k}-u^{\star}\right\|^{2}+\mu(\mathscr{R} \mu-1)\left\|\left(J_{\lambda}^{G_{2}}-I\right) A u_{k}\right\|^{2} .
$$

Since $\mu \in(0,1 / \mathscr{R})$, we obtain

$$
\left\|v_{k}-u^{\star}\right\|^{2} \leq\left\|u_{k}-u^{\star}\right\|^{2}
$$

Now, we show that $\left\{u_{k}\right\}$ is bounded.

$$
\begin{aligned}
\left\|u_{k+1}-u^{\star}\right\|= & \left\|\alpha_{k} f\left(u_{k}\right)+\left(1-\alpha_{k}\right) T_{[k+1]} v_{k}-u^{\star}\right\| \\
\leq & \alpha_{k}\left\|f\left(u_{k}\right)-u^{\star}\right\|+\left(1-\alpha_{k}\right)\left\|T_{[k+1]} v_{k}-u^{\star}\right\| \\
\leq & \alpha_{k}\left\|f\left(u_{k}\right)-f\left(u^{\star}\right)\right\|+\alpha_{k}\left\|f\left(u^{\star}\right)-u^{\star}\right\| \\
& +\left(1-\alpha_{k}\right)\left\|T_{[k+1]]} v_{k}-T_{[k+1]} u^{\star}\right\| \\
\leq & \alpha_{k} \kappa\left\|u_{k}-u^{\star}\right\|+\alpha_{k}\left\|f\left(u^{\star}\right)-u^{\star}\right\| \\
& +\left(1-\alpha_{k}\right)\left\|v_{k}-u^{\star}\right\| \\
= & {\left[1-\alpha_{k}(1-\kappa)\right]\left\|u_{k}-u^{\star}\right\|+\alpha_{k}\left\|f\left(u^{\star}\right)-u^{\star}\right\| } \\
\leq & \max \left\{\left\|u_{k}-u^{\star}\right\|, \frac{\left\|f\left(u^{\star}\right)-u^{\star}\right\|}{1-\kappa}\right\} \\
& \quad \cdots \\
\leq & \max \left\{\left\|u_{0}-u^{\star}\right\|, \frac{\left\|f\left(u^{\star}\right)-u^{\star}\right\|}{1-\kappa}\right\} .
\end{aligned}
$$

Hence, $\left\{u_{k}\right\}$ is bounded, which implies that the sequences $\left\{v_{k}\right\},\left\{f\left(u_{k}\right)\right\}$, and $\left\{T_{[k+1]} v_{k}\right\}$ are also bounded. It follows from nonexpansiveness of $T_{n},(n=1, \cdots M)$, and Lipschitz continuity of $f$ with constant $\kappa$ that

$$
\begin{aligned}
\left\|u_{k+M}-u_{k}\right\|= & \| \alpha_{k+M-1} f\left(u_{k+M-1}\right)+\left(1-\alpha_{k+M-1}\right) T_{[k+M]}\left(v_{k+M-1}\right) \\
& -\alpha_{k-1} f\left(u_{k-1}\right)+\left(1-\alpha_{k-1}\right) T_{[k]}\left(v_{k-1}\right) \| \\
= & \| \alpha_{k+M-1} f\left(u_{k+M-1}\right)-\alpha_{k+M-1} f\left(u_{k-1}\right) \\
& +\alpha_{k+M-1} f\left(u_{k-1}\right)+\left(1-\alpha_{k+M-1}\right) T_{[k]}\left(v_{k-1}\right) \\
& -\left(1-\alpha_{k+M-1}\right) T_{[k]}\left(v_{k-1}\right) \\
& +\left(1-\alpha_{k+M-1}\right) T_{[k+M]}\left(v_{k+M-1}\right)-\alpha_{k-1} f\left(u_{k-1}\right) \\
& +\left(1-\alpha_{k-1}\right) T_{[k]}\left(v_{k-1}\right) \| \\
\leq & \left(\alpha_{k+M-1}\right)\left\|f\left(u_{k+M-1}\right)-f\left(u_{k-1}\right)\right\| \\
& +\left\|f\left(u_{k-1}\right)\right\|\left|\alpha_{k+M-1}-\alpha_{k-1}\right| \\
& +\left(1-\alpha_{k+M-1}\right)\left\|T_{[k+M]}\left(v_{k+M-1}\right)-T_{[k]}\left(v_{k-1}\right)\right\| \\
& +\left\|T_{[k]}\left(v_{k-1}\right)\right\|\left|\alpha_{k-1}-\alpha_{k+M-1}\right|,
\end{aligned}
$$

that is,

$$
\begin{aligned}
\left\|u_{k+M}-u_{k}\right\|= & \kappa\left(\alpha_{k+M-1}\right)\left\|u_{k+M-1}-u_{k-1}\right\| \\
& +\left(1-\alpha_{k+M-1}\right)\left\|v_{k+M-1}-v_{k-1}\right\| \\
& +2\left|\alpha_{k+M-1}-\alpha_{k-1}\right| M_{1},
\end{aligned}
$$

where $M_{1}=\sup \left\{\left\|f\left(u_{k-1}\right)\right\|+\left\|T_{[k]}\left(v_{k-1}\right)\right\|: k \in \mathbb{N}\right\}$. Since, $\mu \in(0,1 / \mathscr{R})$, the operator $J_{\lambda}^{G_{1}}\left[I+\mu A^{*}\left(J_{\lambda}^{G_{2}}-I\right) A\right]$ is average and hence nonexpansive, then we have

$$
\begin{aligned}
\left\|v_{k+M-1}-v_{k-1}\right\|= & \| J_{\lambda}^{G_{1}}\left(u_{k+M-1}+\mu A^{*}\left(J_{\lambda}^{G_{2}}-I\right) A u_{k+M-1}\right) \\
& -J_{\lambda}^{G_{1}}\left(u_{k-1}+\mu A^{*}\left(J_{\lambda}^{G_{2}}-I\right) A u_{k-1}\right) \| \\
\leq & \| J_{\lambda}^{G_{1}}\left[I+\mu A^{*}\left(J_{\lambda}^{G_{2}}-I\right) A\right] u_{k+M-1} \\
& -J_{\lambda}^{G_{1}}\left[I+\mu A^{*}\left(J_{\lambda}^{G_{2}}-I\right) A\right] u_{k-1} \| \\
\leq & \left\|u_{k+M-1}-u_{k-1}\right\| .
\end{aligned}
$$

From (34), (33) becomes

$$
\begin{aligned}
\left\|u_{k+M}-u_{k}\right\| \leq & {\left[1-\alpha_{k+M-1}(1-\kappa)\right]\left\|u_{k+M-1}-u_{k-1}\right\| } \\
& +2\left|\alpha_{k+M-1}-\alpha_{k-1}\right| M_{1},
\end{aligned}
$$

let $\xi_{k}=\alpha_{k+M-1}(1-\kappa), \quad \omega_{k}=2\left|\alpha_{k+M-1}-\alpha_{k-1}\right| M_{1}$, by using Lemma 7 , we conclude that

$$
\lim _{k \rightarrow \infty}\left\|u_{k+M}-u_{k}\right\|=0
$$


Now, we show that $\left\|u_{k}-v_{k}\right\| \longrightarrow 0$ as $k \longrightarrow \infty$. From (29), it follows that

$$
\begin{aligned}
\left\|u_{k+1}-u^{\star}\right\|^{2}= & \left\|\alpha_{k} f\left(u_{k}\right)+\left(1-\alpha_{k}\right) T_{[k+1]} v_{k}-u^{\star}\right\|^{2} \\
\leq & \alpha_{k}\left\|f\left(u_{k}\right)-u^{\star}\right\|^{2}+\left(1-\alpha_{k}\right)\left\|T_{[k+1]} v_{k}-T_{[k+1]} u^{\star}\right\|^{2} \\
\leq & \alpha_{k}\left\|f\left(u_{k}\right)-u^{\star}\right\|^{2}+\left(1-\alpha_{k}\right)\left\|v_{k}-u^{\star}\right\|^{2} \\
\leq & \alpha_{k}\left\|f\left(u_{k}\right)-u^{\star}\right\|^{2}+\left(1-\alpha_{k}\right) \\
& \cdot\left[\left\|u_{k}-u^{\star}\right\|^{2}+\mu(\mathscr{R} \mu-1)\left\|\left(J_{\lambda}^{G_{2}}-I\right) A u_{k}\right\|^{2}\right] .
\end{aligned}
$$

Therefore,

$$
\begin{aligned}
& \mu(-\mathscr{R} \mu+1)\left\|\left(J_{\lambda}^{G_{2}}-I\right) A u_{k}\right\|^{2} \\
& \quad \leq \alpha_{k}\left\|f\left(u_{k}\right)-u^{\star}\right\|^{2}+\left\|u_{k}-u^{\star}\right\|^{2}-\left\|u_{k+1}-u^{\star}\right\|^{2} \\
& \quad=\alpha_{k}\left\|f\left(u_{k}\right)-u^{\star}\right\|^{2}+\left\|u_{k+1}-u_{k}\right\|\left[\left\|u_{k}-u^{\star}\right\|+\left\|u_{k+1}-u^{\star}\right\|\right] .
\end{aligned}
$$
get

Since $(1-\mathscr{R} \mu)>0, \alpha_{k} \longrightarrow 0$, and $\left\|u_{k+1}-u_{k}\right\| \longrightarrow 0$, we

$$
\left\|\left(J_{\lambda}^{G_{2}}-I\right) A u_{k}\right\| \longrightarrow 0, \text { as } k \longrightarrow \infty
$$

Since $\mu \in(0(1 / \mathscr{R}))$ and using (27) and (29), we obtain

$$
\begin{aligned}
\left\|v_{k}-u^{\star}\right\|^{2}= & \left\|J_{\lambda}^{G_{1}}\left(u_{k}+\mu A^{*}\left(J_{\lambda}^{G_{2}}-I\right) A u_{k}\right)-u^{\star}\right\|^{2} \\
= & \left\|J_{\lambda}^{G_{1}}\left(u_{k}+\mu A^{*}\left(J_{\lambda}^{G_{2}}-I\right) A u_{k}\right)-J_{\lambda}^{G_{1}} u^{\star}\right\|^{2} \\
\leq & \left\langle v_{k}-u^{\star}, u_{k}+\mu A^{*}\left(J_{\lambda}^{G_{2}}-I\right) A u_{k}-u^{\star}\right\rangle \\
= & \frac{1}{2}\left[\left\|v_{k}-u^{\star}\right\|^{2}+\left\|u_{k}+\mu A^{*}\left(J_{\lambda}^{G_{2}}-I\right) A u_{k}-u^{\star}\right\|^{2}\right. \\
& \left.-\left\|\left(v_{k}-u^{\star}\right)-\left[u_{k}+\mu A^{*}\left(J_{\lambda}^{G_{2}}-I\right) A u_{k}-u^{\star}\right]\right\|^{2}\right] \\
= & \frac{1}{2}\left[\left\|v_{k}-u^{\star}\right\|^{2}+\left\|u_{k}-u^{\star}\right\|^{2}+\mu(\mathscr{R} \mu-1)\right. \\
& \left.\cdot\left\|\left(J_{\lambda}^{G_{2}}-I\right) A u_{k}\right\|^{2}-\left\|v_{k}-u_{k}-\mu A^{*}\left(J_{\lambda}^{G_{2}}-I\right) A u_{k}\right\|^{2}\right] \\
\leq & \frac{1}{2}\left[\left\|v_{k}-u^{\star}\right\|^{2}+\left\|u_{k}-u^{\star}\right\|^{2}-\left[\left\|v_{k}-u_{k}\right\|^{2}+\mu^{2} \| A^{*}\right.\right. \\
& \left.\left.\cdot\left(J_{\lambda}^{G_{2}}-I\right) A u_{k} \|^{2}-2 \mu\left\langle v_{k}-u_{k}, A^{*}\left(J_{\lambda}^{G_{2}}-I\right) A u_{k}\right\rangle\right]\right] \\
\leq & \frac{1}{2}\left[\left\|v_{k}-u^{\star}\right\|^{2}-\left\|v_{k}-u_{k}\right\|^{2}+\left\|u_{k}-u^{\star}\right\|^{2}\right. \\
& \left.+2 \mu\left\|A\left(v_{k}-u_{k}\right)\right\|\left\|\left(J_{\lambda}^{G_{2}}-I\right) A u_{k}\right\|\right] .
\end{aligned}
$$

Thus, we get

$$
\begin{aligned}
\left\|v_{k}-u^{\star}\right\|^{2} \leq & \left\|u_{k}-u^{\star}\right\|^{2}+2 \mu\left\|A\left(v_{k}-u_{k}\right)\right\|\left\|\left(J_{\lambda}^{G_{2}}-I\right) A u_{k}\right\| \\
-\left\|v_{k}-u_{k}\right\|^{2} &
\end{aligned}
$$

By (37) and (41), we have

$$
\begin{aligned}
\left\|u_{k+1}-u^{\star}\right\|^{2} \leq & \alpha_{k}\left\|f\left(u_{k}\right)-u^{\star}\right\|^{2}+\left(1-\alpha_{k}\right) \\
& \cdot\left[2 \mu\left\|A\left(v_{k}-u_{k}\right)\right\|\left\|\left(J_{\lambda}^{G_{2}}-I\right) A u_{k}\right\|\right. \\
+ & \left.\left\|u_{k}-u^{\star}\right\|^{2}-\left\|v_{k}-u_{k}\right\|^{2}\right] \\
\leq & \alpha_{k}\left\|f\left(u_{k}\right)-u^{\star}\right\|^{2}+2 \mu\left\|A\left(v_{k}-u_{k}\right)\right\| \\
& \cdot\left\|\left(J_{\lambda}^{G_{2}}-I\right) A u_{k}\right\|+\left\|u_{k}-u^{\star}\right\|^{2}-\left\|v_{k}-u_{k}\right\|^{2},
\end{aligned}
$$

that is,

$$
\begin{aligned}
\left\|v_{k}-u_{k}\right\|^{2} \leq & \alpha_{k}\left\|f\left(u_{k}\right)-u^{\star}\right\|^{2}+2 \mu\left\|A\left(v_{k}-u_{k}\right)\right\| \\
& \cdot\left\|\left(J_{\lambda}^{G_{2}}-I\right) A u_{k}\right\|+\left\|u_{k}-u^{\star}\right\|^{2}-\left\|u_{k+1}-u^{\star}\right\|^{2} \\
\leq & \alpha_{k}\left\|f\left(u_{k}\right)-u^{\star}\right\|^{2}+2 \mu\left\|A\left(v_{k}-u_{k}\right)\right\|\left\|\left(J_{\lambda}^{G_{2}}-I\right) A u_{k}\right\| \\
& +\left(\left\|u_{k}-u^{\star}\right\|+\left\|u_{k+1}-u^{\star}\right\|\right)\left(\left\|u_{k+1}-u_{k}\right\|\right) .
\end{aligned}
$$

Since $\alpha_{k} \longrightarrow 0,\left\|u_{k+1}-u_{k}\right\| \longrightarrow 0,\left\|\left(J_{\lambda}^{G_{2}}-I\right) A u_{k}\right\| \longrightarrow 0$ as $k \longrightarrow \infty$, we get

$$
\lim _{k \rightarrow \infty}\left\|v_{k}-u_{k}\right\|=0
$$

We recognized that the following relation holds:

$$
\begin{aligned}
& u_{k+M}-u_{k}=u_{k+M}-T_{[k+M]}\left(v_{k+M-1}\right) \\
& +T_{[k+M]}\left(v_{k+M-1}\right)-T_{[k+M]}\left(u_{k+M-1}\right) \\
& +T_{[k+M]}\left(u_{k+M-1}\right)-T_{[k+M]} T_{[k+M-1]}\left(v_{k+M-2}\right) \\
& + \\
& \cdots \\
& +T_{[k+M]}{ }^{\cdots} \cdots \circ T_{[k+2]}\left(u_{k+1}\right)-T_{[k+M]} \circ \cdots \circ T_{[k+2]}\left(v_{k+1}\right) \\
& +T_{[k+M]} \circ \cdots \circ T_{[k+2]}\left(v_{k+1}\right)-T_{[k+M]} \circ \cdots \circ T_{[k+1]}\left(u_{k}\right)
\end{aligned}
$$

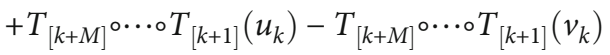

$$
\begin{aligned}
& +T_{[k+M]}{ }^{\cdots} \cdots \circ T_{[k+1]}\left(v_{k}\right)-u_{k} \text {. }
\end{aligned}
$$


By Iterative Scheme 8 , we can easily see that $\| u_{k+1}$ $T_{[k+1]} v_{k} \| \longrightarrow 0$ as $k \longrightarrow \infty$. From (44) and nonexpansiveness of $T_{n}(n=1,2, \cdots, M)$, it follows that

$$
\begin{aligned}
& \left\|u_{k+M}-T_{[k+M]}\left(v_{k+M-1}\right)\right\| \longrightarrow 0 \\
& \left\|T_{[k+M]}\left(v_{k+M-1}\right)-T_{[k+M]}\left(u_{k+M-1}\right)\right\| \longrightarrow 0 \\
& \left\|T_{[k+M]}\left(u_{k+M-1}\right)-T_{[k+M]} \circ T_{[k+M-1]}\left(v_{k+M-2}\right)\right\| \longrightarrow 0
\end{aligned}
$$

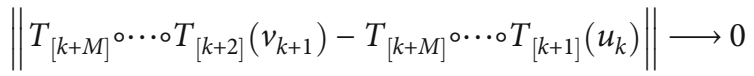

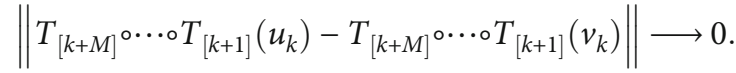

By using (36) and (45), we conclude that

$$
\lim _{k \rightarrow \infty}\left\|T_{[k+M]} \circ T_{[k+M-1]} \circ \cdots \circ T_{[k+2]} \circ T_{[k+1]}\left(v_{k}\right)-u_{k}\right\| \longrightarrow 0 .
$$

Now, using (47) and (44), we write

$$
\begin{aligned}
& \left\|T_{[k+M]} \circ \quad T_{[k+M-1]}{ }^{\circ \cdots \circ} T_{[k+1]}\left(v_{k}\right)-v_{k}\right\| \\
& \leq \| T_{[k+M]} \circ T_{[k+M-1]}{ }^{\circ \cdots \circ T_{[k+1]}\left(v_{k}\right)-u_{k} \|} \\
& +\left\|v_{k}-u_{k}\right\| \longrightarrow 0 \text {, }
\end{aligned}
$$

as $k \longrightarrow \infty$, that is,

$$
\left\|T_{[k+M]} \circ T_{[k+M-1]} \circ \cdots \circ T_{[k+1]}\left(v_{k}\right)-u_{k}\right\| \longrightarrow 0 \text { as } k \longrightarrow \infty .
$$

Boundedness of $\left\{v_{k}\right\}$ implies that there exists a subsequence $\left\{v_{k_{i}}\right\}$ of $\left\{v_{k}\right\}$, converging weakly to $w$. Because the collection of mappings $\left\{T_{n}: 1 \leq n \leq M\right\}$ is finite, we can say for some integer $K \in\{1,2, \cdots, N\}$

$$
T_{\left[k_{n}\right]} \equiv T_{K}, \quad \forall n \geq 1 .
$$

Thus, from (49), we have

$$
\left\|T_{[n+M]} \circ T_{[n+M-1]^{0}} \cdots \circ T_{[n+1]}\left(v_{k_{n}}\right)-v_{k_{n}}\right\| \longrightarrow 0 .
$$

Therefore, using Lemma 6, we conclude that

$$
w \in \operatorname{Fix}\left(T_{[n+M]} \cdots T_{[n+1]}\right) .
$$

Thus, by the assumptions of Condition $C$, we have $w \in \cap{ }_{n=1}^{M} \operatorname{Fix}\left(T_{n}\right)$. On the other hand,

$$
\begin{aligned}
& v_{k_{i}}=J_{\lambda}^{G_{1}}\left[u_{k_{i}}+\mu A^{*}\left(J_{\lambda}^{G_{2}}-I\right) A u_{k_{i}}\right] \\
& \frac{u_{k_{i}}+\mu A^{*}\left(J_{\lambda}^{G_{2}}-I\right) A u_{k_{i}}}{} \in\left(I+\lambda G_{1}\right) v_{k_{i}} \\
& \frac{u_{k_{i}}-v_{k_{i}}+\mu A^{*}\left(J_{\lambda}^{G_{2}}-I\right) A u_{k_{i}}}{\lambda} \in G_{1}\left(v_{k_{i}}\right) .
\end{aligned}
$$

We know that the graph of a maximal monotone operator is weakly strongly closed; hence, by taking $i \longrightarrow \infty$ and using (37) and (44), we get

$$
0 \in G_{1}(w)
$$

Since $\left\{u_{k}\right\},\left\{v_{k}\right\}$ have the same asymptotical behaviour, $\left\{A u_{k_{i}}\right\}$ converges weakly to $A w$. Therefore, by (39), the nonexpansive property of $J_{\lambda}^{G_{2}}$ and Lemma 6 , we get $0 \in G_{2}$ $(A w)$. Thus, $w \in \cap_{n=1}^{M} \operatorname{Fix}\left(T_{n}\right) \cap \Xi$.

Now, we need to show that $\limsup _{k \rightarrow \infty}\left\langle f(\bar{v})-\bar{v}, u_{k}-\bar{v}\right\rangle \leq 0$, where $\bar{v}=P_{\cap_{n=1}^{M} \operatorname{Fix}\left(T_{n}\right) \cap \Xi} f(\bar{v})$.

We have

$$
\begin{aligned}
\limsup _{k \rightarrow \infty}\left\langle f(\bar{v})-\bar{v}, u_{k}-\bar{v}\right\rangle & =\limsup _{k \rightarrow \infty}\left\langle f(\bar{v})-\bar{v}, T_{[k+1]} v_{k}-\bar{v}\right\rangle \\
& \leq\langle f(\bar{v})-\bar{v}, w-\bar{v}\rangle \leq 0,
\end{aligned}
$$

since $\bar{v} \in \cap_{n=1}^{M} \operatorname{Fix}\left(T_{n}\right) \cap \Xi$.

Finally, we show that $x_{k} \longrightarrow \bar{v}$

$$
\begin{aligned}
\left\|u_{k+1}-\bar{v}\right\|^{2}= & \left\|\alpha_{k} f\left(u_{k}\right)+\left(1-\alpha_{k}\right) T_{[k+1]}\left(v_{k}\right)-\bar{v}\right\|^{2} \\
= & \left\langle\alpha_{k} f\left(u_{k}\right)+\left(1-\alpha_{k}\right) T_{[k+1]}\left(v_{k}\right)-\bar{v}, u_{k+1}-\bar{v}\right\rangle \\
= & \alpha_{k}\left\langle f\left(u_{k}\right)-\bar{v}, u_{k+1}-\bar{v}\right\rangle+\left(1-\alpha_{k}\right) \\
& \cdot\left\langle T_{[k+1]} v_{k}-\bar{v}, u_{k+1}-\bar{v}\right\rangle \leq \alpha_{k}\left\langle f\left(u_{k}\right)-\bar{v}, u_{k+1}-\bar{v}\right\rangle \\
& +\left(1-\alpha_{k}\right)\left\langle v_{k}-\bar{v}, u_{k+1}-\bar{v}\right\rangle \\
\leq & \alpha_{k}\left\langle f\left(u_{k}\right)-f(\bar{v}), u_{k+1}-\bar{v}\right\rangle+\alpha_{k}\left\langle f\left(u_{k}\right)-\bar{v}, u_{k+1}-\bar{v}\right\rangle \\
& +\left(1-\alpha_{k}\right)\left\langle v_{k}-\bar{v}, u_{k+1}-\bar{v}\right\rangle \\
\leq & \frac{\alpha_{k}}{2}\left\{\left\|f\left(u_{k}\right)-f(\bar{v})\right\|^{2}+\left\|u_{k+1}-\bar{v}\right\|^{2}\right\} \\
& +\alpha_{k}\left\langle f(\bar{v})-\bar{v}, u_{k+1}-\bar{v}\right\rangle+\frac{\left(1-\alpha_{k}\right)}{2} \\
& \cdot\left\{\left\|u_{k}-\bar{v}\right\|^{2}+\left\|u_{k+1}-\bar{v}\right\|^{2}\right\} \\
\leq & \frac{1}{2}\left[1-\alpha_{k}\left(1-\kappa^{2}\right)\right]\left\|u_{k+1}-\bar{v}\right\|^{2}+\frac{\left(1-\alpha_{k}\right)}{2} \\
& \cdot\left\|u_{k}-\bar{v}\right\|^{2}+\frac{\alpha_{k}}{2}\left\|u_{k+1}-\bar{v}\right\|^{2} \\
& +\alpha_{k}\left\langle f(\bar{v})-\bar{v}, u_{k+1}-\bar{v}\right\rangle,
\end{aligned}
$$


which implies that

$$
\begin{aligned}
\left\|u_{k+1}-\bar{v}\right\|^{2} \leq & {\left[1-\alpha_{k}\left(1-\kappa^{2}\right)\right]\left\|u_{k+1}-\bar{v}\right\|^{2} } \\
& +2 \alpha_{k}\left\langle f(\bar{v})-\bar{v}, u_{k+1}-\bar{v}\right\rangle .
\end{aligned}
$$

From Lemma 7 and (55), we conclude that $u_{k} \longrightarrow \bar{v}$ and from $\left\|v_{k}-u_{k}\right\| \longrightarrow 0, v_{k}-w \in \cap_{n=1}^{M} \operatorname{Fix} T_{n} \cap \Xi$, and $u_{k} \longrightarrow \bar{v}$ as $k \longrightarrow \infty$, we achieve that $\bar{v}=w$. This completes the proof.

\section{Consequences}

Suppose $\mathscr{C}$ and $\mathscr{D}$ are closed convex subsets of Hilbert spaces $\mathscr{H}_{1}$ and $\mathscr{H}_{2}$, respectively. Then, find $u \in \mathscr{H}_{1}$ such that

$$
u \in \mathscr{C} \text { and } A u \in \mathscr{D} \text {, }
$$

is called the split feasibility problem (SFP), where $A$ : $\mathscr{H}_{1} \longrightarrow \mathscr{H}_{2}$ is a bounded linear operator. Byrne [9] introduced the $\mathscr{C} \mathscr{D}$ algorithm to approximate the solution of (58):

$$
u_{k+1}=P_{\mathscr{C}}\left(u_{k}+\mu A^{*}\left(P_{\mathscr{D}}-I\right) A u_{k}\right)
$$

where $P_{\mathscr{C}}$ and $P_{\mathscr{D}}$ are orthogonal projections onto $\mathscr{C}$ and $\mathscr{D}$, respectively.

The split common fixed-point problem (SCFPP) is an extension of Problem (58), which has been widely investigated in the present scenario. The SCFPP is the inverse problem design to search a vector in a fixed-point set so that its image under a bounded linear operator corresponds to other fixed-point set, that is, find $u \in \mathscr{H}_{1}$ such that

$$
u=W(u) \text { and } A u=V(A u)
$$

where $W: \mathscr{H}_{1} \longrightarrow \mathscr{H}_{1}$ and $W: \mathscr{H}_{2} \longrightarrow \mathscr{H}_{2}$ are nonexpansive mappings. By putting $W=P_{\mathscr{C}}$ and $W=P_{\mathscr{D}}$, in (59), we can have an iterative scheme, which converges to the solution of SCFPP.

We denote the solution set of SFP (58) and SCFPP (60) by $\Psi$, and $\Omega$, respectively. The following corollaries are given as consequences of Theorem 12.

Corollary 13. Let $\mathscr{C}$ and $\mathscr{D}$ be two closed convex subsets of Hilbert spaces $\mathscr{H}_{1}$ and $\mathscr{H}_{2}$, respectively. Let $A: \mathscr{H}_{1} \longrightarrow \mathscr{H}_{2}$ be a bounded linear operator and $f: \mathscr{H}_{1} \longrightarrow \mathscr{H}_{1}$ be a contraction mapping with constant $\kappa \in(0,1)$. Let $T_{n}: \mathscr{H}_{1} \longrightarrow$ $\mathscr{H}_{1},(n=1,2, \cdots, M)$, be a finite collection of nonexpansive mappings satisfying the condition $C$ such that $\cap_{n=1}^{M} \operatorname{Fix}\left(T_{n}\right)$ $\cap \Psi \neq 0$. Let $\mathscr{R}$ be the spectral radius of $A^{\star} A$, where $A^{\star}$ is the adjoint of $A$ such that $\mu \in(0,1 / \mathscr{R})$ and $\left\{\alpha_{k}\right\}$ be a sequence in $(0,1)$ with $\lim _{k \rightarrow \infty} \alpha_{k}=0, \sum_{k=1}^{\infty} \alpha_{k}=\infty$, and $\sum_{k=1}^{\infty}\left|\alpha_{k}-\alpha_{k-1}\right|<$ $\infty$. Then, the iterative sequences $\left\{v_{k}\right\}$ and $\left\{u_{k}\right\}$ generated by Iterative Scheme 8 with $J_{\lambda}^{G_{1}}=P_{\mathscr{C}}$ and $J_{\lambda}^{G_{2}}=P_{\mathscr{D}}$ converge

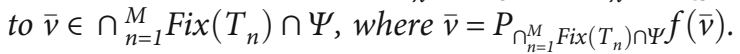

TABLE 1: Computation of iterative sequences of Iterative Scheme 8 for the choices of parameters $\lambda=1 / 4$ and $\alpha_{n}=1 / 3 k$ and different initial points $u_{0}=5$ and $u_{0}=-3$.

\begin{tabular}{lcccc}
\hline$\lambda=1 / 4$ & & $u_{0}=5$ & & $u_{0}=-3$ \\
No. iter. & $v_{k}$ & $u k+1$ & $v_{k}$ & $u k+1$ \\
\hline 0 & 3.5 & -0.48502 & -0.25 & -1.831663 \\
1 & -1.613765 & -1.034683 & -1.623747 & -0.726077 \\
2 & -1.26012 & -0.956449 & -0.794558 & -0.748519 \\
3 & -0.967337 & -0.926583 & -0.8113989 & -0.775985 \\
4 & -0.944937 & -0.989916 & -0.8319989 & -1.037605 \\
5 & -0.992437 & -0.968237 & -1.028204 & -0.984733 \\
6 & -0.976178 & -0.948843 & -0.998550 & -0.964922 \\
7 & -0.961632 & -0.996486 & -0.973792 & -0.991042 \\
8 & -0.997364 & -0.980131 & -0.993282 & -0.977974 \\
9 & -0.985098 & -0.968597 & -0.983481 & -0.966999 \\
10 & -0.976448 & -0.995792 & -0.975249 & -0.996349 \\
11 & -0.986844 & -0.984495 & -0.997262 & -0.984711 \\
12 & -0.984495 & -0.975650 & -0.988533 & -0.975811 \\
13 & -0.981737 & -0.996719 & -0.981858 & -0.996662 \\
14 & -0.997539 & -0.997635 & -0.997496 & -0.987612 \\
\hline
\end{tabular}

Corollary 14. Let $\mathscr{H}_{1}$ and $\mathscr{H}_{2}$ be two real Hilbert spaces and A : $\mathscr{H}_{1} \longrightarrow \mathscr{H}_{2}$ be a bounded linear operator. Assume that $G_{1}: \mathscr{H}_{1} \longrightarrow 2^{\mathscr{H}_{1}}$ and $G_{2}: \mathscr{H}_{2} \longrightarrow 2^{\mathscr{H}_{2}}$ are maximal monotone operators and $f: \mathscr{H}_{1} \longrightarrow \mathscr{H}_{1}$ is a $\kappa$-contraction mapping with constant $\kappa \in(0,1)$. Let $T_{n}: \mathscr{H}_{1} \longrightarrow \mathscr{H}_{1}$, $(n=1,2, \cdots, M)$, be a finite collection of nonexpansive mappings satisfying the condition $C$ such that $\cap_{n=1}^{M} F i x\left(T_{n}\right) \cap \Omega$ $\neq 0$. Let $\mathscr{R}$ be spectral radius of $A^{\star} A$, where $A^{\star}$ is the adjoint of $A$ such that $\mu \in(0,1 / \mathscr{R})$ and $\left\{\alpha_{k}\right\}$ be a sequence in $(0,1)$ with $\lim _{n \rightarrow \infty} \alpha_{k}=0, \sum_{k=1}^{\infty} \alpha_{k}=\infty$, and $\sum_{k=1}^{\infty}\left|\alpha_{k}-\alpha_{k-1}\right|<\infty$. Then, the iterative sequences $\left\{v_{k}\right\}$ and $\left\{u_{k}\right\}$ obtained from Iterative Scheme 8 with $J_{\lambda}^{G_{1}}=W$ and $J_{\lambda}^{G_{2}}=V$ converge to $\bar{v} \in \cap_{n=1}^{M}$ Fix $\left(T_{n}\right) \cap \Omega$, where $\bar{v}=P_{\cap_{n=1}^{M} F i x\left(T_{n}\right) \cap \Omega} f(\bar{v})$.

Remark 15. If we take $T_{1}=T_{2}=\cdots T_{M}=T$, a nonexpansive mapping, then we can obtain the iterative scheme and its convergence theorem for the common solution of $S_{p} V I P$ and a nonexpansive mapping $T$, studied in [17].

At last, we illustrate the convergence analysis of the proposed iterative scheme with the help of the following numerical example.

\section{Numerical Example}

Let $\mathscr{H}_{1}=\mathscr{H}_{2}=\mathbb{R}$ and $G_{1}: \mathscr{H}_{1} \longrightarrow \mathscr{H}_{1}$ defined as $G_{1}(u)=2$ $(u+1)$ and $G_{2}: \mathscr{H}_{2} \longrightarrow \mathscr{H}_{2}$ defined as $G_{2}(u)=-(4 / 5) u+$ $(12 / 5)$. For $\lambda=1 / 4$, we compute the resolvents of $G_{1}$ and $G_{2}$ as

$$
\begin{aligned}
& J_{\lambda}^{G_{1}}(u)=\left[I+\lambda G_{1}\right]^{-1}(u)=\frac{2}{3} u-\frac{1}{3}, \\
& J_{\lambda}^{G_{2}}(u)=\left[I+\lambda G_{2}\right]^{-1}(u)=\frac{5}{4} u-\frac{3}{4} .
\end{aligned}
$$


It can be easily seen that, here, $\Xi=\{-1\}$. Further, let $T_{1}, T_{2}$, and $T_{3}: \mathscr{H}_{1} \longrightarrow \mathscr{H}_{1}$ are three nonexpansive mappings, defined by

$$
\begin{aligned}
& T_{1}(u)=\sin (u+1)-1, \\
& T_{2}(u)=\frac{-u-3}{2}, \\
& T_{3}(u)=\frac{\cos (\pi u)+u}{2}
\end{aligned}
$$

such that

$$
\operatorname{Fix}\left(T_{1}\right) \cap \operatorname{Fix}\left(T_{2}\right) \cap \operatorname{Fix}\left(T_{3}\right)=\{-1\} .
$$

Let $f: \mathscr{H}_{1} \longrightarrow \mathscr{H}_{1}$ be a contraction mapping defined as $f(u)=u / 2$ and $A$ be a bounded linear operator defined as $A u=-3 u$ with adjoint operator $A^{*}$ such that $\|A\|=\left\|A^{*}\right\|=3$.

Since $\mu \in(0,1 / 9)$ and $\alpha_{k} \in(0,1)$, so we choose $\mu=1 / 18$ and $\alpha_{k}=1 / 3 k$; then, the sequences $\left\{v_{k}\right\}$ and $\left\{u_{k}\right\}$ generated by the iterative scheme are evaluated as

$$
\begin{aligned}
v_{k} & =J_{\lambda}^{G_{1}}\left[u_{k}+\mu A^{*}\left(J_{\lambda}^{G_{2}}-I\right) A u_{k}\right]=\frac{9}{12} u_{k}-\frac{3}{12}, \\
u_{k+1} & =\alpha_{k} f\left(u_{k}\right)+\left(1-\alpha_{k}\right) T_{[k+1]} v_{k} \\
& =\frac{1}{6(k+1)} u_{k}+\left[1-\frac{1}{3(k+1)}\right] T_{[k+1]} v_{k}
\end{aligned}
$$

or for some positive integer $a \geq 0$, and $M=3$, we can write

$$
u_{k+1}= \begin{cases}\frac{u_{k}}{6(k+1)}+\left[1-\frac{1}{3(k+1)}\right]\left[\sin \left(v_{k}+1\right)-1\right], & \text { if } k=3 a+1, \\ \frac{u_{k}}{6(k+1)}+\left[1-\frac{1}{3(k+1)}\right] \frac{-v_{k}-3}{2}, & \text { if } k=3 a+2, \\ \frac{u_{k}}{6(k+1)}+\left[1-\frac{1}{3(k+1)}\right] \frac{\cos \left(\pi v_{k}\right)+v_{k}}{2}, & \text { if } k=3 a .\end{cases}
$$

From Table 1, we conclude that for two arbitrary different initial points $u_{0}=5$ and $u_{0}=-3$, the sequences $\left\{v_{k}\right\}$ and $\left\{u_{k}\right\}$ converge approximately to a point $u^{\star}=-1 \in \cap_{n=1}^{M}$ Fix $\left(T_{n}\right) \cap \Xi$.

\section{Data Availability}

No data were used to support the findings of the study.

\section{Conflicts of Interest}

No potential conflict of interest is reported by the authors.

\section{Authors' Contributions}

All authors read and approved the final manuscript.

\section{References}

[1] A. Farajzadeh, P. Chuasuk, A. Kaewcharoen, and M. Mursaleen, "An iterative process for a hybrid pair of generalized I-asymptotically nonexpansive single-valued mappings and generalized nonexpansive multi-valued mappings in Banach spaces," Carpathian Journal of Mathematics, vol. 34, no. 1, pp. 31-45, 2018.

[2] G. Marino and H. K. Xu, "A general iterative method for nonexpansive mappings in Hilbert spaces," Journal of Mathematical Analysis and Applications, vol. 318, no. 1, pp. 4352, 2006.
[3] W. R. Mann, "Mean value methods in iteration," Proceedings of American Mathematical Society, vol. 4, no. 3, pp. 506-510, 1953.

[4] A. Moudafi, "Viscosity approximation methods for fixedpoints problems," Journal of Mathematical Analysis and Applications, vol. 241, no. 1, pp. 46-55, 2000.

[5] H. K. Xu, "Viscosity approximation methods for nonexpansive mappings," Journal of Mathematical Analysis and Applications, vol. 298, no. 1, pp. 279-291, 2004.

[6] Y. Censor, A. Gibali, and S. Reich, "Algorithms for the split variational inequality problem," Numerical Algorithms, vol. 59, no. 2, pp. 301-323, 2012.

[7] A. Moudafi, "Split monotone variational inclusions," Journal of Optimization Theory and Applications, vol. 150, no. 2, pp. 275-283, 2011.

[8] C. Byrne, Y. Censor, A. Gibali, and S. Reich, "Weak and strong convergence of algorithms for split common null point problem," Journal of Nonlinear and Convex Analysis, vol. 13, pp. 759-775, 2012.

[9] C. Byrne, "Iterative oblique projection onto convex sets and the split feasibility problem," Inverse Problems, vol. 18, no. 2, pp. 441-453, 2002.

[10] L.-C. Ceng and Q. Yuan, "Strong convergence of a new iterative algorithm for split monotone variational inclusion problems," Mathematics, vol. 7, no. 2, p. 123, 2019.

[11] M. Dilshad, M. Akram, and I. Ahmad, "Algorithms for split common null point problem without pre-existing estimation of operator norm," Journal of Mathematical Inequalities, 2020. 
[12] A. Farajzadeh, M. Mursaleen, and A. Shafie, "On mixed vector equilibrium problems," Azerbaijan Journal of Mathematics, vol. 6, no. 2, pp. 87-102, 2016.

[13] J. K. Kim, M. Salahuddin, and W. H. Lim, "General nonconvex split variational inequality problems," Korean Journal of Mathematics, vol. 25, no. 4, pp. 469-481, 2017.

[14] A. Moudafi, "The split common fixed-point problem for demicontractive mappings," Inverse Problems, vol. 26, no. 5, article 055007, 2010.

[15] W. Takahashi and J.-C. Yao, "Strong convergence theorems by hybrid methods for the split common null point problem in Banach spaces," Fixed Point Theory and Applications, vol. 2015, no. 1, Article ID 324, 2015.

[16] M. Tian and B. N. Jiang, "Viscosity approximation methods for a class of generalized split feasibility problems with variational inequalities in Hilbert space," Numerical Functional Analysis and Optimization, vol. 40, no. 8, pp. 902-923, 2019.

[17] K. R. Kazmi and S. H. Rizvi, "An iterative method for split variational inclusion problem and fixed point problem for a nonexpansive mapping," Optimization Letters, vol. 8, no. 3, pp. 1113-1124, 2014.

[18] J. Puangpee and S. Suantai, "A new algorithm for split variational inclusion and fixed point problems in Banach spaces," Computational and Mathematical Methods, vol. 2, no. 2, article e1078, 2020.

[19] H. Che and M. Li, "Solving split variational inclusion problem and fixed point problem for nonexpansive semigroup without prior knowledge of operator norms," Mathematical Problems in Engineering, vol. 2015, Article ID 408165, 9 pages, 2015.

[20] M. Abbas, Y. Ibrahim, A. R. Khan, and M. De la Sen, "Split variational inclusion problem and fixed point problem for a class of multivalued mappings in CAT(0) spaces," Mathematics, vol. 7, no. 8, p. 749, 2019.

[21] M. Dilshad, A. H. Siddiqi, R. Ahmad, and F. A. Khan, "An iterative algorithm for a common solution of a split variational inclusion problem and fixed point problem for nonexpansive semigroup mappings," in Industrial Mathematics and Complex Systems. Industrial and Applied Mathematics, P. Manchanda, R. Lozi, and A. Siddiqi, Eds., Springer, Singapore, 2017.

[22] P. Lohawech, A. Kaewcharoen, and A. Farajzadeh, "Algorithms for the common solution of the split variational inequality problems and fixed point problems with applications," Journal of Inequalities and Applications, vol. 2018, no. 1, Article ID 358, 2018.

[23] K. Siriyan and K. Atid, "Algorithm method for solving the split general system of variational inequalities problem and fixed point problem of nonexpansive mapping with application," Mathematical Methods in the Applied Sciences, vol. 41, no. 17, pp. 7766-7788, 2018.

[24] Y. Yao, Y.-C. Liou, and J.-C. Yao, "Iterative algorithms for the split variational inequality and fixed point problems under nonlinear transformations," The Journal of Nonlinear Sciences and Applications, vol. 10, no. 2, pp. 843-854, 2017.

[25] G. Crombez, "A hierarchical presentation of operators with fixed points on Hilbert spaces," Numerical Functional Analysis and Optimization, vol. 27, no. 3-4, pp. 259-277, 2006.

[26] G. Crombez, "A geometrical look at iterative methods for operators with fixed points," Numerical Functional Analysis and Optimization, vol. 26, no. 2, pp. 157-175, 2005.
[27] H. H. Bauschke and P. L. Combettes, Convex Analysis and Monotone Operator Theory in Hilbert Spaces, Springer, New York, 2011.

[28] G. Lopez, V. Martin-Marquez, and H. K. Xu, "Iterative algorithm for the multi-sets feasibility problems," in Biomedical Mathematics, Promising Direction in Imaging Therapy Planning and Inverse Problems, Y. Censor, M. Jiang, and G. Wang, Eds., pp. 243-279, Medical Physics Publishing, Madison, 2010.

[29] K. Goebel and W. A. Kirk, Topics on Fixed Point Theory, Cambridge University Press, Cambridge, 2009. 